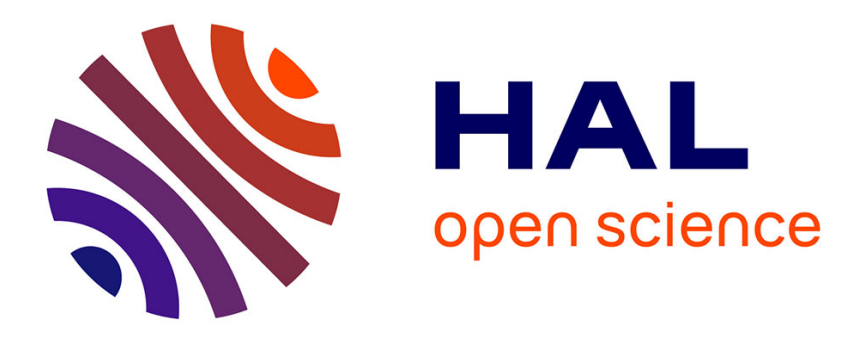

\title{
Modelling of sound generation by turbulent reacting flows
}

\author{
Christophe Bailly, Christophe Bogey, Sébastien M. Candel
}

\section{To cite this version:}

Christophe Bailly, Christophe Bogey, Sébastien M. Candel. Modelling of sound generation by turbulent reacting flows. International Journal of Aeroacoustics, 2010, 9, pp.461-489. 10.1260/1475-472X.9.45.461 . hal-00566028

\section{HAL Id: hal-00566028 https://hal.science/hal-00566028}

Submitted on 8 Jun 2012

HAL is a multi-disciplinary open access archive for the deposit and dissemination of scientific research documents, whether they are published or not. The documents may come from teaching and research institutions in France or abroad, or from public or private research centers.
L'archive ouverte pluridisciplinaire HAL, est destinée au dépôt et à la diffusion de documents scientifiques de niveau recherche, publiés ou non, émanant des établissements d'enseignement et de recherche français ou étrangers, des laboratoires publics ou privés. 


\title{
Modelling of sound generation by turbulent reacting flows
}

\author{
Christophe Bailly', Christophe Bogey ${ }^{1} \&$ Sébastien Candel ${ }^{2}$ \\ 1 Laboratoire de Mécanique des Fluides et d'Acoustique, \\ Ecole Centrale de Lyon \& UMR CNRS 5509 \\ 36 avenue Guy de Collongue, 69134 Ecully cedex, France. \\ 2Laboratoire EM2C Ecole Centrale Paris \& CNRS UPR 288, \\ Grande Voie des Vignes, 92295 Chatenay-Malabry cedex, France.
}

Submitted June 1, 2009; Revised September 17, 2009; Accepted January 12, 2010

\begin{abstract}
The two components of combustion noise, namely the direct noise induced by heat release fluctuations in the flow and the indirect noise caused by acceleration of entropy perturbations through mean flow gradients resulting in acoustic radiation, are examined by considering different types of inhomogeneous wave equations deduced from the balance equations of fluid dynamics. This is accomplished by adapting acoustic analogies initially derived by Lighthill (1952), Phillips (1960) and Lilley (1972) for non-reactive flows to the reacting case. This reformulation is based on a standard set of assumptions borrowed from combustion. It is shown that a consistent formulation of a wave operator and its associated source terms is required.
\end{abstract}

\section{INTRODUCTION}

Among the many noise sources associated with aero-engines the contribution of combustion noise is not easy to assess. In a typical noise source breakdown of commercial aircraft this component only represents a few percent of the total noise emitted at take-off [43]. However this contribution lies in the low frequency range and it is only weakly attenuated by liners inside the nacelle duct. It is also known that the separation between combustion and jet noise components is difficult to make in practice, as shown by Hoch et al. [41], Muthukrishnan et al. [59], Parthasarathy et al. [62] or more recently by Harper-Bourne et al. [39] and Blacodon [6]. Noise induced by combustion actually originates from two distinct processes. The first, designated as core noise or direct combustion noise is associated with the rate of heat release fluctuations

Corresponding author: Christophe Bailly, christophe.bailly@ec-lyon.fr 
inside the combustor. The second, indirect combustion noise is generated when entropy spots essentially temperature fluctuations produced by combustion are accelerated through an inhomogeneous mean flow field radiating acoustic waves. This mechanism occurs for example in the turbine blade rows when this assembly is traversed by fluctuations generated by combustion. The increase of fluctuations in the flow at the nozzle exit may reach intensity levels of about $10 \%$ with strengthened velocity correlations as pointed out by Plett \& Summerfield [67] or by Cumpsty \& Marble. [22] As a result, a dipolar lip noise can dominate the radiated far field at low exhaust velocities, as argued by Ffowcs Williams \& Gordon [34], in a frequency range similar to that of subsonic noise. Some of the first identifications of entropy noise as a potential noise source is due to Candel [13] and Marble \& Candel [54] who demonstrated this mechanism by considering the convection of entropy waves in a one-dimensional compact nozzle. Models of the interaction between entropy fluctuations and turbine blade rows were developed during the same period by Pickett [65] and by Cumpsty et al . $[23,24]$ using actuator disk theory in idealized configurations. Other theoretical investigation were established in the framework of an acoustic analogy to include acoustic-flow interactions, like refraction and scattering of sound waves, refer to Lilley [52], Morfey [57] and to Ffowcs Williams \& Howe [32] among others. A natural link can indeed be drawn between the study of temperature effects in aeroacoustics and indirect combustion noise.

Combustion noise is relatively less well documented than other topics in aeroacoustics like aerodynamic noise $[8,20,38,81]$. The effort in computational aeroacoustics $[3,17,84]$ has also been focused on non-reactive flows. An extended effort has been made to examine the mechanisms of combustion instabilities in which noise generated by combustion is closely coupled by the acoustics of the system inducing large amplitude oscillations with many detrimental effects. Many studies also consider the passive and active control of these processes and applications to high performance devices like gas turbines, aero- and rocket engines. This question has a long history with an early instability criterion devised by Rayleigh [72]. Some historical references may be found in Feldman [30] or in Pierce [66]. Combustion instabilities are not considered in the present article, and the reader can refer to Ducruix et al. [27], Dowling \& Morgans [26], Culick [21] or to Candel et al. [12] for recent comprehensive reviews. Initial experiments and theoretical studies have shown that noise generated by turbulent flames is characterized by a broadband spectrum $[16,75,77,78]$ without discrete frequency peaks when there is no instability due to an acoustic coupling or to an external forcing. The spectrum is comparable with that of the fine-scale mixing noise of subsonic turbulent jets. In the simplest configurations, noise is generated by the unsteady rate of heat release induced by combustion which leads to a monopolar and isotropic sound radiation in the low frequency range, for Strouhal numbers less than 0.5, as demonstrated in the early experiments work carried out by Thomas and Williams [82] and in the theoretical results derived by Strahle [79,80] from Lighthill's analogy [49].

The present paper attempts a synthesis of combustion noise based on the most common aeroacoustic theories, which can be derived by starting from the balance of fluid dynamics and extracting an inhomogeneous wave equation. The next section 
gathers the balance equations for a reacting gas mixture. Direct and indirect combustion noise are then discussed by extending Lighthill's analogy in Section 3. Noise generated by a spherically expanding laminar flame is also briefly revisited. Flow-acoustic interactions are illustrated in Section 4 and an extension of Phillips' equation is derived for reacting flows. The low Mach number limit is also considered. Lilley's equation is finally derived for combustion noise in Section 5. An hybrid approach based on linearized Euler's equations and a discussion of the set of acoustic perturbation equations [11] used in several recent studies of combustion noise are also reported in this section. A derivation of an alternative form of the energy equation, $[12,68]$ of classical wave equations of Phillips [64] and Lilley [53], as well as of a wave equation associated with linearized Euler's equations are carried out in the appendixes.

\section{REACTIVE FLOW CONSERVATION EOUATIONS}

The equations which govern a gas-phase reacting flow can be found in several textbooks $[61,68,85]$ and only some of the key expressions need to be included in what follows. The balance of mass, momentum and energy can be written in the following form

$$
\begin{gathered}
\frac{d \rho}{d t}+\rho \nabla \cdot \boldsymbol{u}=0 \\
\rho \frac{d \boldsymbol{u}}{d t}=-\nabla p+\nabla \cdot \boldsymbol{\tau} \\
\rho \frac{d e_{t}}{d t}=\nabla \cdot(-\boldsymbol{q}-p \boldsymbol{u}+\boldsymbol{\tau} \cdot \boldsymbol{u})
\end{gathered}
$$

where $d / d t=\partial_{t}+\boldsymbol{u} \cdot \nabla$ is the material derivative. The previous expressions involve the density $\rho$, velocity $\boldsymbol{u}$, pressure $p$, viscous stress tensor $\tau$, total energy $e_{t}=e+$ $(1 / 2) u^{2}$ and heat flux $\boldsymbol{q}$. For simplicity, there is no external force, neither radiation nor external heat sources. Conservation of chemical species $k$ is expressed by

$$
\rho \frac{d Y_{k}}{d t}=\dot{\omega}_{k}-\nabla \cdot \boldsymbol{J}_{k}
$$

where $Y_{k}$ is the mass fraction of species $k, \dot{\omega}_{k}$ is the reaction rate, which is determined from Arrhenius-like laws and $\boldsymbol{J}_{k}$ is the diffusion flux resulting from the diffusion velocities of the different species in the mixture, $\boldsymbol{J}_{k}=\rho Y_{k} \boldsymbol{v}_{k}$ where $\boldsymbol{v}_{k}$ is the diffusion velocity of species $k$. It is assumed that all species behave like perfect gases so that the mixture follows a perfect gas law

$$
p=\rho r T \quad 1 /\left(c_{p} T\right)=(\gamma-1) / c^{2} \quad c^{2}=\gamma r T=\frac{\gamma p}{\rho}
$$


where $\gamma=c_{p} / c_{v}$ is the ratio of specific heats at constant pressure and volume, $T$ is the temperature, $c$ is the local speed of sound and $r=R / W$ is the specific gas constant, with $R$ the universal gas constant and $W$ the mean molar weight of the mixture. The last two relations in (5) will be used to simplify equations in what follows. The balance of energy (3) can also be written using the enthapy $h$ of the mixture, which yields

$$
\rho \frac{d h}{d t}=\frac{d p}{d t}-\nabla \cdot \boldsymbol{q}+\boldsymbol{\tau}: \nabla \boldsymbol{u}
$$

and a straightforward calculation reported in Appendix A leads to the following equivalent expression

$$
\frac{d \rho}{d t}=\frac{1}{c^{2}} \frac{d p}{d t}+\frac{\gamma-1}{c^{2}}\left[-\dot{\omega}_{T}-\nabla \cdot(\lambda \nabla T)+\rho \sum_{k} Y_{k} c_{p, k} \boldsymbol{v}_{k} \cdot \nabla T-\boldsymbol{\tau}: \nabla \boldsymbol{u}\right]-\rho \frac{d}{d t}(\ln r)(7)
$$

where $\dot{\omega}_{T}$ is the heat release per unit volume, expressed in watts per cubic meter, produced by combustion. The reader familiar with acoustic analogies will recognize in Eq. (7) the combination $d \rho / d t-\left(1 / c^{2}\right) d p / d t$ useful for deriving wave equations.

\section{COMBUSTION NOISE THROUGH LIGHTHILL'S ANALOGY}

Lighthill's theory of aerodynamic noise [49] is now considered as the starting point of aeroacoustics as a research topic. Rather than directly solving fluid dynamics equations (1) to (3) to calculate aerodynamic sound, an equivalent problem is obtained in the form of a wave equation with a source term. The solution is then derived using an appropriate Green function. Applying the material derivative to mass conservation equation and substracting the divergence of the momentum conservation equation, the resulting expression $d(1) / d t-\nabla \cdot(2)=0$ yields,

$$
\frac{\partial^{2} \rho}{\partial t^{2}}-\nabla^{2} p=\nabla \cdot \nabla \cdot(\rho \boldsymbol{u u}-\boldsymbol{\tau})
$$

This expression is completed to obtain the Dalembertian operator on the left-hand side, which yields for the density

$$
\frac{\partial^{2} \rho}{\partial t^{2}}-c_{\infty}^{2} \nabla^{2} \rho=\nabla \cdot \nabla \cdot(\rho \boldsymbol{u u}-\boldsymbol{\tau})+\nabla^{2}\left(p-c_{\infty}^{2} \rho\right)
$$

or equivalently for the pressure,

$$
\frac{1}{c_{\infty}^{2}} \frac{\partial^{2} p}{\partial t^{2}}-\nabla^{2} p=\nabla \cdot \nabla \cdot(\rho \boldsymbol{u u}-\boldsymbol{\tau})-\frac{\partial^{2}}{\partial t^{2}}\left(\rho-\frac{1}{c_{\infty}^{2}} p\right)
$$


In these two expressions (8) and (9), the velocity $c_{\infty}$ is an arbitrary constant, as recently illustrated by Peake [63] for the propagation of a normal shock wave. In many practical applications and especially in jet noise analysis, $c_{\infty}$ is taken to be the speed of sound in the medium at rest or at infinity, This gives a physical meaning to the wave operator $[3,9]$ which describes propagation in the medium surrounding the free jet. The first term on the right-hand side of equation (9) represents the contribution of the Reynolds and viscous stresses. Combustion noise is included in the second source term, a point which is nicely illustrated by Ffowcs Williams [31] or Lilley [51,52]. By noting that density is a function of two variables, the pressure and the specific enthalpy $\rho=$ $\rho(p, h)$ for instance, the differentiation of $\rho$ leads to

$$
d \rho=\left.\frac{d \rho}{d p}\right|_{h} d p+\left.\frac{d \rho}{d h}\right|_{p} d h=\frac{1}{c^{2}} d p-\rho \frac{\gamma-1}{c^{2}} d h
$$

for a perfect gas. Therefore, the residue of $d \rho-\left(1 / c^{2}\right) d p$ corresponds to the heat added to the fluid particule per unit mass. This result will be found later in a more formal way. It is convenient to introduce at this step an excess density $\rho_{e}$ as proposed by Morfey [57],

$$
\rho_{e}=\left(\rho-\rho_{\infty}\right)-\frac{1}{c_{\infty}^{2}}\left(p-p_{\infty}\right)
$$

Indeed, an estimator of the deviation from the adiabatic relation $d \rho=d p / c_{\infty}^{2}$, which holds for acoustical perturbations in a medium at rest, is then obtained. The integral solution of Eq. (9) can therefore be written as a convolution product of the source term, given by

$$
S=\nabla \cdot \nabla \cdot(\rho \boldsymbol{u u}-\boldsymbol{\tau})-\frac{\partial^{2} \rho_{e}}{\partial t^{2}}
$$

with the free-space Green function $G_{0}(\boldsymbol{x}, t)=\delta\left(t-x / c_{\infty}\right) /(4 \pi x)$, that is

$$
p(\boldsymbol{x}, t)=\frac{1}{4 \pi} \int_{V} S\left(\boldsymbol{y}, t-\frac{\xi}{c_{\infty}}\right) \frac{d \boldsymbol{y}}{r} \quad \text { with } \quad \boldsymbol{\xi}=\boldsymbol{x}-\boldsymbol{y}
$$

The integration is taken over the region $V$ containing sources, i.e. for which $S \neq 0$, and at the retarded time $t-\xi / c_{\infty}$ corresponding to the travelling time from the source $\boldsymbol{y}$ to the observer $\boldsymbol{x}$, as illustrated in Fig. 1. As underlined in the two reviews by Crighton [20] and by Ffowcs Williams, [19] subtle formulations of this integral solution can be developed in the far field as $x \gg y$, or also by using properties of the convolution product and applying the differential operator to either the Green function or the source term. A physical interpretation of this solution (11) will be discussed later in this section. 


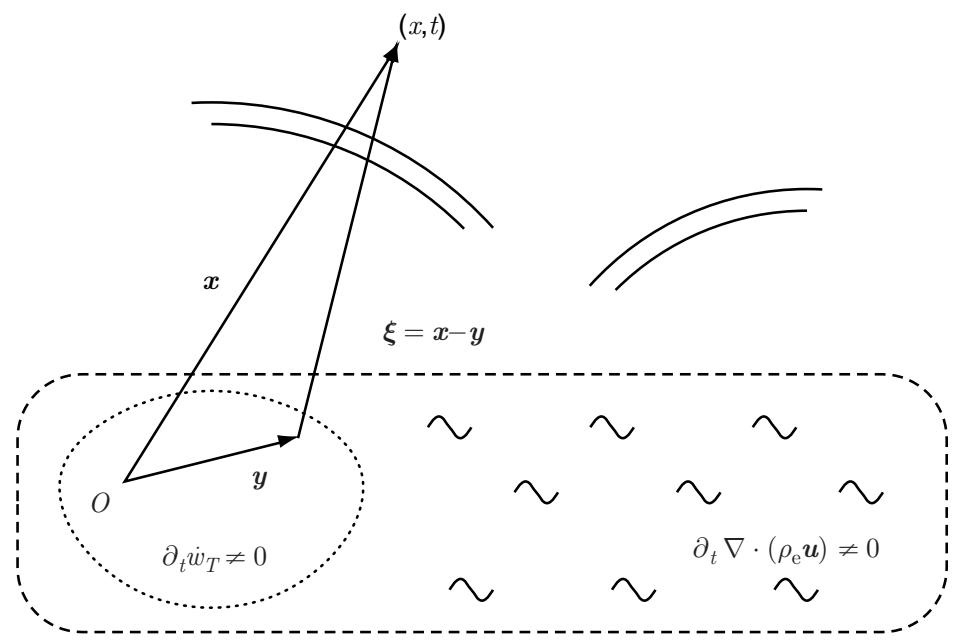

Source volume $V$

Figure 1: $\quad$ Sketch illustrating Lighthill's integral solution given by Eq. (14). By neglecting aerodynamic noise, the source term in the volume $V$ basically represents the contribution of the direct combustion noise associated with the unsteady rate of heat release $\partial_{t} \dot{\omega}_{T}$ and of the entropy noise associated with the term $\partial_{t} \nabla \cdot\left(\rho_{e} \boldsymbol{u}\right) \neq 0$.

In what follows, a canonical and general expression of Lighthill's analogy for a reactive turbulent flow is derived without any approximation. To this end, the main source term $\partial^{2} \rho_{e} / \partial t^{2}$ is developed using relation (7). The time derivative of excess density is first written as $[19,31,57]$

$$
\frac{\partial \rho_{e}}{\partial t}=\frac{d \rho_{e}}{d t}-\boldsymbol{u} \cdot \nabla \rho_{e}=\frac{d \rho_{e}}{d t}-\frac{\rho_{e}}{\rho} \frac{d \rho}{d t}-\nabla \cdot\left(\rho_{e} \boldsymbol{u}\right)
$$

by using the balance of mass (1). Substituting $\rho_{e}$ by its definition (10) gives

$$
\frac{\partial \rho_{e}}{\partial t}=\frac{\rho_{\infty}}{\rho} \frac{d \rho}{d t}-\frac{1}{c_{\infty}^{2}} \frac{d p}{d t}+\frac{1}{c_{\infty}^{2} \rho}\left(p-p_{\infty}\right) \frac{d \rho}{d t}-\nabla \cdot\left(\rho_{e} \boldsymbol{u}\right)
$$

Using the balance of energy Eq. (7), one gets

$$
\begin{aligned}
\frac{\partial \rho_{e}}{\partial t}= & \frac{\rho_{\infty}}{\rho} \frac{\gamma-1}{c^{2}}\left(-\dot{\omega}_{T}-\nabla \cdot(\lambda \nabla T)+\rho \sum_{k} Y_{k} c_{p, k} \boldsymbol{v}_{k} \cdot \nabla T-\boldsymbol{u}: \nabla \boldsymbol{\tau}\right) \\
& -\rho_{\infty} \frac{d}{d t}(\ln r)-\nabla \cdot\left(\rho_{e} \boldsymbol{u}\right)-\frac{1}{c_{\infty}^{2}}\left(1-\frac{\rho_{\infty} c_{\infty}^{2}}{\rho c^{2}}\right) \frac{d p}{d t}+\frac{p-p_{\infty}}{\rho c_{\infty}^{2}} \frac{d \rho}{d t}
\end{aligned}
$$


Finally, and without introducing any approximation, the source term $S$ appearing in the integral solution (11) can be written as follows

$$
\begin{aligned}
S & =\nabla \cdot \nabla \cdot(\rho \boldsymbol{u} \boldsymbol{u}-\boldsymbol{\tau}) \\
& +\frac{\partial}{\partial t}\left[\frac{\rho_{\infty}}{\rho} \frac{\gamma-1}{c^{2}}\left(\dot{\omega}_{T}+\nabla \cdot(\lambda \nabla T)-\rho \sum_{k} Y_{k} c_{p, k} \boldsymbol{v}_{k} \cdot \nabla T+\boldsymbol{u}: \nabla \boldsymbol{\tau}\right)+\rho_{\infty} \frac{d}{d t}(\ln r)\right] \\
& +\frac{1}{c_{\infty}^{2}} \frac{\partial}{\partial t}\left[\left(1-\frac{\rho_{\infty} c_{\infty}^{2}}{\rho c^{2}}\right) \frac{d p}{d t}-\frac{p-p_{\infty}}{\rho} \frac{d \rho}{d t}\right] \\
& +\nabla \cdot \frac{\partial}{\partial t}\left(\rho_{e} \boldsymbol{u}\right)
\end{aligned}
$$

This is an exact reformulation of the fluid dynamics equations for a reacting flow. The two first terms on the right-hand side of Eq. (13) are associated with aerodynamic noise as already mentioned, and as it can also be seen by noting that the viscous term is generally very small with respect to the double divergence of the Reynolds stress tensor $\nabla \cdot \nabla \cdot(\rho \boldsymbol{u u})$, especially for turbulent flows at high Reynolds numbers $[46,56]$. This expression is the starting point of the derivation of the classical eighth power scaling law obtained by Lighthill [49] for subsonic jet noise. The terms in the second line represent various types of processes. The main contribution is expected to come from the fluctuating heat release directly produced by combustion, namely

$$
\frac{\partial}{\partial t}\left[\frac{\rho_{\infty}}{\rho} \frac{(\gamma-1)}{c^{2}} \dot{\omega}_{T}\right]=\frac{\partial}{\partial t}\left(\frac{\rho_{\infty}}{\rho} \frac{\dot{\omega}_{T}}{c_{p} T}\right)
$$

where Eq. (5) has been used. Terms appearing in the third line are mainly linked to the flow inhomogeneities with respect to the propagation medium. This flow-acoustic interaction disappears for iso-baric combustion. The last term in the fourth line represents acceleration of density inhomogeneities, and represents the main contribution to indirect combustion noise [19].

Classical assumptions such as a constant molecular weight of the mixture, negligeable diffusion flux terms and a quasi-isobaric flow at low Mach number $\mathrm{M}=u / c_{\infty} \ll 1$, can be used to simplify the source term expression (13). Noise is then generated by the unsteady heat release rate induced by combustion, and the far field pressure is given by

$$
p(\boldsymbol{x}, t) \simeq \frac{1}{4 \pi x} \frac{\partial}{\partial t} \int_{V} \frac{\rho_{\infty}}{\rho} \frac{(\gamma-1)}{c^{2}} \dot{\boldsymbol{\omega}}_{T}\left(\boldsymbol{y}, t-\frac{\xi}{c_{\infty}}\right) d \boldsymbol{y} \quad x \gg y
$$


where the origin is taken inside the source volume $S$, see Fig. 1. Moreover, assuming a heat ratio independent of temperature provides $\rho_{\infty} /\left(\rho c^{2}\right)=1 / c_{\infty}^{2}$ for isobaric combustion one finds

$$
p(\boldsymbol{x}, t) \simeq \frac{(\gamma-1)}{4 \pi c_{\infty}^{2} x} \frac{\partial}{\partial t} \int_{V} \dot{\boldsymbol{\omega}}_{T}\left(\boldsymbol{y}, t-\frac{\xi}{c_{\infty}}\right) d \boldsymbol{y} \quad x \gg y
$$

Expression (15) can be found in the work of Strahle [79,80] for instance. It is also discussed by Dowling [19]. Other approximations can be found in the literature $[31,33,83]$. Scaling rules can also be derived $[16,40,75,79]$ for the thermo-acoustic efficiency $\eta_{t a}$, defined as the ratio between the radiated acoustic power and the thermal power released in the flow, with typically $\eta_{t a} \sim 10^{-6}-10^{-5}$ for combustion systems in the absence of instabilities. The noise mechanism involved in Eq. (15) represents a monopolar source, which is usually compact indicating that the typical correlation length of the source is small with respect to the acoustic wavelength, and as a result, the variation of the retarded time can be neglected during integration, which implies that $t-\xi / c_{\infty}$ is replaced by $t-x / c_{\infty}$.

\subsection{An example of direct combustion noise}

One of the fundamental experiment dealing with combustion noise was carried out by Thomas \& Williams. [82] A mixture of air and ethylene is confined within a soap bubble, and combustion is ignited by a spark. A spherical flame front of radius $r_{f}(t)$ slowly propagates, as shown in Fig. 2. The quasi-steady pressure on either side of the flame is assumed constant, yielding $\rho_{\infty} T_{\infty}=\rho_{b} T_{b}$. Noise generated by combustion is measured by a microphone in a small anechoic room whereas the laminar flame is monitored through an optical Schlieren system.

The current volume of burnt gas is $v_{b}=(4 / 3) \pi r_{f}^{3}$ and the initial volume occupied by these fresh mixture was $v_{b} / E$, where $E=\rho_{\infty} / \rho_{b}$ is the density ratio of the unburnt to burnt gas or the volumetric expansion ratio, with typically $7 \leq E \leq 10$ for hydrocarbons. The volume increase is thus given by $\delta V=(4 / 3) \pi r_{f}^{3}(1-1 / E)$, and the rate of volume variation during flame propagation is

$$
\dot{V}=\frac{\delta V}{\delta t}=\frac{E-1}{E} 4 \pi r_{f}^{2} \frac{d r_{f}}{d t}
$$

To numerically estimate the noise radiated by combustion, the authors used a compact monopolar source model to obtain the following expression for the far field pressure,

$$
p(\boldsymbol{x}, t) \simeq \frac{\rho_{\infty}}{4 \pi x} \frac{d \dot{V}}{d t}\left(t-x / c_{\infty}\right)
$$




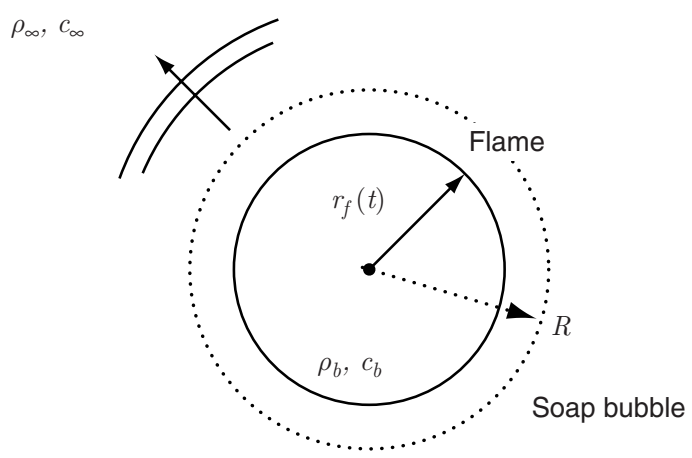

Figure 2: $\quad$ Noise generated by a spherically expanding flame, refer to Thomas \& Williams. ${ }^{82}$ A mixture of air and ethylene is used as premixed combustible gas within a soap bubble, yielding basically $\mathrm{C}_{2} \mathrm{H}_{4}+3 \mathrm{O}_{2} \rightarrow$ $2 \mathrm{CO}_{2}+2 \mathrm{H}_{2} \mathrm{O}$. Density and speed of sound of the ambient medium as well as the mixture are $\rho_{\infty}$ and $c_{\infty}$ whereas those of burn gas are denoted $\rho_{b}$ and $c_{b}$.

The pressure signal deduced from Eq. (16) through the measurement of $r_{f}$ and the pressure detected by the microphone were found to be in good agreement [82]. Expression (16) can also be retrieved directly from Lighthill's analogy (14), as proposed by Howe [42]. The heat release can indeed be written as follows

$$
\dot{\omega}_{T} \simeq \rho_{\infty} c_{p}\left(T_{b}-T_{\infty}\right) \dot{m}_{v} \delta\left(x-r_{f}\right) \quad \text { where } \quad \dot{m}_{v}=\frac{1}{E} 4 \pi r_{f}^{2} \frac{d r_{f}}{d t}
$$

is the volumetric rate of consumption of the combustible, $\dot{m}_{v}=d\left(v_{\mathrm{b}} / E\right) / d t$. After rearrangement and by using Eq. (5), the pressure in the far-field is given by

$$
p(\boldsymbol{x}, t) \simeq \frac{\rho_{\infty}}{4 \pi x}(E-1) \frac{d \dot{m}_{v}}{d t}\left(t-x / c_{\infty}\right)
$$

in agreement with expression (16). Introducing the flame velocity $u_{l}$ relative to fresh mixture, defined as $u_{l}=(1 / E) d r_{f} / d t$ with $\dot{m}_{v}=A u_{l}$ and where $A$ is the flame surface, the acoustic power can be written as

$$
W_{a} \simeq \frac{\rho_{\infty}}{4 \pi c_{\infty}}(1-E)^{2} u_{l}^{2} \overline{\left(\frac{d A}{d t}\right)^{2}}
$$

As a result, the leading term provides a scaling law $W_{a} \sim u_{l}^{4}$ corresponding to the noise emitted by a compact monopole source as expected. 


\subsection{Measurement of combustion noise sources}

The acoustic signature of flames can be used as a non intrusive diagnostic tool to obtain the combustion heat release distribution by solving an inverse problem [70]. Optical techniques are however more widely employed, as exemplified in a reference experimental work by Hurle et al. [44]. Experimentally, the volumetric rate of combustion $\dot{m}_{v}$ which appears in Eq. (17) is found to be directly proportional to the light-emission intensity $I$ radiated by excited radicals like $\mathrm{CH}^{*}, \mathrm{C}_{2}$ or $\mathrm{OH}^{*}$ which are only present in the reaction region [12]. Thus, the relation $\partial_{t} \dot{m}_{v}=k \partial_{t} I$ where $k$ is a constant and the integral solution (17) can then be applied to estimate combustion noise $[74,76]$. This question is discussed for example by Schuller et al. [73] in the case of noise generated by an excited impinging premixed jet flame. The present identification approach however rests on the assumption that the main noise source term is associated with the unsteady rate of heat release. Bui et al. [11] have recently investigated this assumption, and have shown that this term does not fully account for combustion noise radiation in the high frequency range, at Strouhal numbers exceeding 0.5.

\section{COMBUSTION NOISE THROUGH PHILLIPS' ANALOGY}

\subsection{Flow-acoustic interactions}

Lighthill's analogy yielding the integral solution (11) with the source term (13), is attractive since the noise generation problem consists of determining equivalent sources embedded in a uniform medium of sound speed $c_{\infty}$ at rest. Therefore, interactions between the turbulent flow and the sound field generated by this flow, such as convection, refraction or diffraction, are necessarily included in the source term.

To illustrate this point, consider the case of a turbulent flow surrounded by a uniform mean flow $U_{\infty} x_{1}$, as shown in Fig. 3, the primitive formulation of Lighthill's analogy given by Eq. (8) and a source term $S=\partial_{x i} \partial_{x j}\left(\rho u_{i} u_{j}\right)$ associated with aerodynamic noise. Introducing the following decomposition $\rho=\rho_{\infty}+\breve{\rho}$ and $u_{i}=U_{\infty} \delta_{1 i}+\breve{u}_{i}$ in the source term, one gets

$$
\rho u_{i} u_{j}=\left(\rho_{\infty}+\breve{\rho}\right)\left(U_{\infty} \delta_{1 i}+\breve{u}_{i}\right)\left(U_{\infty} \delta_{1 j}+\breve{u}_{j}\right)
$$

It is straightforward to show that Lighthill's equation can be exactly written in the form [2]

$$
\frac{\partial^{2} \breve{\rho}}{\partial t^{2}}-c_{\infty}^{2} \nabla^{2} \breve{\rho}=\frac{\partial^{2}}{\partial x_{i} \partial x_{j}}\left(\rho \breve{u}_{i} \breve{u}_{j}\right)-2 U_{\infty} \frac{\partial^{2} \breve{\rho}}{\partial t \partial x_{1}}-U_{\infty}^{2} \frac{\partial^{2} \breve{\rho}}{\partial x_{1}^{2}}
$$

The last two terms on the right-hand side can be combined with the first term on the left-hand side to retrieve a convected wave operator

$$
\left(\partial_{t}+U_{\infty} \partial_{x_{1}}\right)^{2}-c_{\infty}^{2} \nabla^{2}
$$




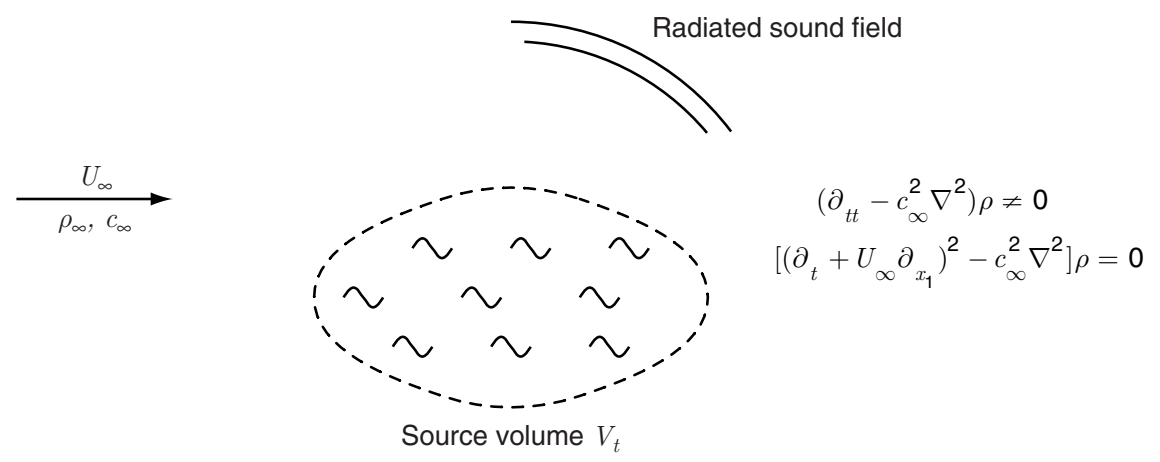

Figure 3: Noise generated by a turbulent flow surrounded by a uniform mean flow.

which governs sound propagation in this particular case. In other words, the terms describing mean flow - acoustic interactions are contained in the source term and density fluctuations can not be neglected when considering acoustic terms, as emphasized by Lilley [50] for instance. These linear terms can be estimated via a compressible simulation of the turbulent flow, and Lighthill's equation can be solved using the whole volume between the turbulent source region $V_{t}$ and the observer, since the term $\left(\partial_{t t}-c_{\infty}^{2} \nabla^{2}\right) \rho$ is never zero. This enlightening but academic exercice has been carried out by Bogey et al. [9] to recover vortex pairing noise generated by a mixing layer. It is worth noting at this point that the computation of the compressible linear source terms requires the knowledge of the acoustic field, that is the solution of the problem itself [3]. In order to conserve a localized source volume, the turbulent volume $V_{t}$ in the present case, flow-acoustic interactions must be removed from the source term, by simply using the suitable convected wave equation. However the derivation of a wave equation governing sound propagation in an arbitrary mean flow remains a difficult and controversial task in aeroacoustics.

\subsection{Phillips' equation for reacting flows}

The first significant step to include flow-acoustic interaction in the wave operator is due to Phillips [64], and the corresponding derivation is reproduced in Appendix B for a non reacting flow, see Eq. (46). For a reacting flow, the balance of mass is combined with the balance of energy (7) to give

$$
\begin{aligned}
\frac{\partial u_{j}}{\partial x_{j}} & =-\frac{1}{\rho} \frac{d \rho}{d t} \\
& =-\frac{1}{\gamma p} \frac{d p}{d t}-\frac{\gamma-1}{\rho c^{2}}\left(-\dot{\omega}_{T}-\nabla \cdot(\lambda \nabla T)+\rho \sum_{k} Y_{k} c_{p, k} \boldsymbol{v}_{k} \cdot \nabla T-\boldsymbol{\tau}: \nabla \boldsymbol{u}\right)+\frac{d}{d t}(\ln r)
\end{aligned}
$$


Following Phillips' developements for the non reacting case [64], the inhomogeneous convected wave equation may be written as

$$
\begin{aligned}
\frac{d^{2} \pi}{d t^{2}}-\frac{\partial}{\partial x_{i}}\left(c^{2} \frac{\partial \pi}{\partial x_{i}}\right)= & \frac{\partial u_{i}}{\partial x_{j}} \frac{\partial u_{j}}{\partial x_{i}}+\frac{d}{d t}\left(\frac{\gamma-1}{\rho c^{2}} \dot{\omega}_{T}\right) \\
& +\frac{d}{d t}\left[\frac{\gamma-1}{\rho c^{2}}\left(\nabla \cdot(\lambda \nabla T)-\rho \sum_{k} Y_{k} c_{p, k} \boldsymbol{v}_{k} \cdot \nabla T+\boldsymbol{\tau}: \nabla \boldsymbol{u}\right)\right] \\
& -\frac{\partial}{\partial x_{i}}\left(\frac{1}{\rho} \frac{\partial \tau_{i j}}{\partial x_{j}}\right)+\frac{d^{2}}{d t^{2}}(\ln r)
\end{aligned}
$$

where the variable $\pi$ is linked to the logarithm of the pressure by the relation $\pi=$ $(1 / \gamma) \ln \left(p / p_{\infty}\right)$. This wave equation was introduced by Chiu \& Summerfield [14] and Kotake [48] for combustion noise, and can also be found in the exact present form in Poinsot and Veynante [68] or Candel et al. [12]. It is often assumed that molecular transport terms can be neglected for sound generation $[46,48,56]$, which thus leads to a simplified version of Eq. (19),

$$
\frac{d^{2} \pi}{d t^{2}}-\frac{\partial}{\partial x_{i}}\left(c^{2} \frac{\partial \pi}{\partial x_{i}}\right) \simeq \frac{\partial u_{i}}{\partial x_{j}} \frac{\partial u_{j}}{\partial x_{i}}+\frac{d}{d t}\left(\frac{\gamma-1}{\rho c^{2}} \dot{\omega}_{T}\right)+\frac{d^{2}}{d t^{2}}(\ln r)
$$

The first term on the right-hand side represents aerodynamic noise, and density no longer appears in this source term [20]. As a result, the ambiguity mentioned in the previous section about flow-acoustic interactions is apparently removed by the convected wave operator. Unfortunately, as shown by Lilley [53], all these interactions are not included on the left-hand side of this equation, and flow effects on acoustics are still contained in the linear part of the first source term. The second source term represents combustion noise through the unsteady heat release, and the third term corresponds to the noise generated by the fluctuation of the molecular weight of the mixture during combustion $[12,83]$.

A key point regarding Eq. (20) is the interpretation of the wave operator in the framework of an acoustic analogy, as already discussed for Lighthill's equation. A first step consists in identifying a wave equation governing sound propagation, such as the Dalembertian for Lighthill's analogy or the convected wave operator (18). The wave operator associated with Phillips' equation is now replaced by

$$
\mathcal{L}_{1}=\frac{\bar{D}^{2}}{\bar{D} t^{2}}-\nabla \cdot\left[\bar{c}^{2}(\boldsymbol{x}) \nabla\right]
$$

where $\bar{D} / \bar{D} t=\partial_{t}+\bar{U} \cdot \nabla$ is the material derivative along the mean flow, and by noting that for small perturbations, $\pi^{\prime} \simeq p^{\prime} /\left(\gamma p_{\infty}\right)$. A classical assumption in combustion is 
also to assume a low Mach number flow, which can be used to remove the convective part of the material derivative, and the ambiguity on the aerodynamic source term. In this way, the wave operator is reduced to

$$
\mathcal{L}_{2}=\frac{\partial^{2}}{\partial t^{2}}-\nabla \cdot\left[\bar{c}^{2}(\boldsymbol{x}) \nabla\right]
$$

or equivalently in a more familiar form, see Goldstein [38] or Pierce [66], to

$$
\frac{1}{\bar{c}^{2}(\boldsymbol{x})} \mathcal{L}_{2}=\frac{1}{\bar{c}^{2}(\boldsymbol{x})} \frac{\partial^{2}}{\partial t^{2}}-\bar{\rho}(\boldsymbol{x}) \nabla \cdot\left[\frac{1}{\bar{\rho}(\boldsymbol{x})} \nabla\right]
$$

This last equation is widely used for underwater and atmospheric long-range sound propagation in an inhomogeneous medium at rest. Linear contributions of source terms in Eq. (13), attributed to flow - acoustic interactions, are now included in the wave operator $\mathcal{L}_{2}$ and can be explicitly calculated. There is however no known analytical Green function associated with this wave operator, and so no integral formulation giving the far field pressure.

Kaltenbacher et al. [45] considered Phillips' equation (20) with the simplified wave operator given by Eq. (21) to develop a numerical approach in the low Mach number limit for combustion noise. These is used to develop a finite-element method of solution in order to tackle noise generation problems in complex geometries.

\subsection{Low mach number limit of Phillip's equation}

In the low Mach number limit, allowing to approximate the material derivative by the time derivative only, Eq. (20) becomes

$$
\frac{\partial}{\partial t}\left(\frac{1}{\gamma p} \frac{\partial p}{\partial t}\right)-\frac{\partial}{\partial x_{i}}\left(\frac{1}{\rho} \frac{\partial p}{\partial x_{i}}\right) \simeq \frac{\partial u_{i}}{\partial x_{j}} \frac{\partial u_{j}}{\partial x_{i}}+\frac{\partial}{\partial t}\left(\frac{\gamma-1}{\rho c^{2}} \dot{\omega}_{T}\right)+\frac{\partial^{2}}{\partial t^{2}}(\ln r)
$$

Another interpretation of Phillips' equation can then be proposed by forming the Dalembertian on the left-hand side of this equation, which yields after some rearrangements,

$$
\begin{aligned}
\frac{1}{c_{\infty}^{2}} \frac{\partial^{2} p}{\partial t^{2}}-\nabla^{2} p & \simeq \frac{1}{c_{\infty}^{2}} \frac{\partial}{\partial t}\left[\left(1-\frac{\rho_{\infty} c_{\infty}^{2}}{\rho c^{2}}\right) \frac{\partial p}{\partial t}\right]-\nabla \cdot\left[\left(1-\frac{\rho_{\infty}}{\rho}\right) \nabla p\right] \\
& +\rho_{\infty} \frac{\partial u_{i}}{\partial x_{j}} \frac{\partial u_{j}}{\partial x_{i}}+\frac{\partial}{\partial t}\left(\frac{\rho_{\infty}}{\rho} \frac{\gamma-1}{c^{2}} \dot{\omega}_{T}\right)+\rho_{\infty} \frac{\partial^{2}}{\partial t^{2}}(\ln r)
\end{aligned}
$$

As mentioned by Candel et al. [12], this expression is already derived by Howe [42], see his Eq. (6.4.2), by neglecting the fluctuations of the molar composition of 
the mixture during combustion. Eq. (22) can be compared to Lighthill's Eq. (13) in the low Mach number flow limit, and by recalling that molecular transports have also been neglected to obtain Eq. (22). Thus, the three source terms in the second line of Eq. (22), that is aerodynamic noise, combustion noise and the noise induced by variations of the mean molecular weight of the mixture, can be easily identified. The first source term in the first line is also present in Eq. (13). This is however not the case for the second term.

At this step, it can be observed that the indirect combustion noise is represented by the two first source terms on the right-hand side in Eq. (22). By comparison with Eq. (13) obtained from Lighthill's analogy, acceleration of excess density through the term $\nabla \cdot \partial_{t}\left(\rho_{e} \boldsymbol{u}\right)$ seems rearranged in a different manner. This result can be explained by noting that in Lighthill's analogy, the low Mach number limit is applied to a source term including linear propagation terms. Generation and propagation are however better separated in Phillips's approach, and it thus seems more consistent to use Eq. (22).

\section{LILLEY'S EOUATION}

Following the developments included in Appendix C, it is straightforward to obtain Lilley's equation for a reacting gas mixture. This equation writes

$$
\begin{aligned}
& \frac{d}{d t}\left[\frac{d^{2} \pi}{d t^{2}}-\frac{\partial}{\partial x_{i}}\left(c^{2} \frac{\partial \pi}{\partial x_{i}}\right)\right]+2 \frac{\partial u_{i}}{\partial x_{j}} \frac{\partial}{\partial x_{i}}\left(c^{2} \frac{\partial \pi}{\partial x_{j}}\right)=-2 \frac{\partial u_{i}}{\partial x_{j}} \frac{\partial u_{j}}{\partial x_{k}} \frac{\partial u_{k}}{\partial x_{i}}+\frac{d^{2}}{d t^{2}}\left(\frac{\gamma-1}{\rho c^{2}} \dot{\omega}_{T}\right) \\
& +\frac{d^{2}}{d t^{2}}\left[\frac{\gamma-1}{\rho c^{2}}\left(\nabla \cdot(\lambda \nabla T)-\rho \sum_{k} Y_{k} c_{p, k} \boldsymbol{v}_{k} \cdot \nabla T+\boldsymbol{\tau}: \nabla \boldsymbol{u}\right)\right] \\
& \quad+2 \frac{\partial u_{i}}{\partial x_{j}} \frac{\partial}{\partial x_{i}}\left(\frac{1}{\rho} \frac{\partial \tau_{i j}}{\partial x_{i}}\right)-\frac{d}{d t}\left[\frac{\partial}{\partial x_{i}}\left(\frac{1}{\rho} \frac{\partial \tau_{i j}}{\partial x_{j}}\right)\right]+\frac{d^{3}}{d t^{3}}(\ln r)
\end{aligned}
$$

and the meaning of the different terms on the right-hand side of this equation has already been discussed in previous sections. This equation plays a fundamental role in aeroacoustics by providing a way to separate sound propagation effects from sound generation, as explained by Doak [25] and Goldstein [38] among others.

\subsection{Interpretation of the wave operator}

To begin with Lilley's equation, it is again important to correctly interpret the wave operator on the left-hand side of Eq. (23). And a key point to do that is first to recognize that the Euler equations linearized around an arbitrarily steady flow govern sound propagation in this flow $[7,25,38,66]$. From these equations given in Appendix D, an homogeneous wave equation can only be derived for a sheared mean flow, that is $\bar{u}_{i}=$ $\bar{u}_{1}\left(x_{2}, x_{3}\right) \delta_{1 i}$ for the mean velocity, whereas $\bar{\rho}=\bar{\rho}\left(x_{2}, x_{3}\right)$ and $\bar{c}=\bar{c}\left(x_{2}, x_{3}\right)$ are only 
functions of transverse coordinates, the mean pressure then being constant. Noting $\bar{D}_{t} \equiv \partial_{t}+\bar{u}_{1} \partial_{x_{1}}$ the material derivative along the mean flow, linearized Euler's equations reduce to

$$
\left\{\begin{array}{l}
\bar{D}_{t} \rho^{\prime}+\nabla \cdot\left(\bar{\rho} \boldsymbol{u}^{\prime}\right)=0 \\
\bar{D}_{t}\left(\bar{\rho} \boldsymbol{u}^{\prime}\right)+\bar{\rho} \boldsymbol{u}^{\prime} \cdot \nabla \overline{\boldsymbol{u}}+\nabla p^{\prime}=\boldsymbol{S}^{m} \\
\bar{D}_{t} p^{\prime}+\gamma \bar{p} \nabla \cdot \boldsymbol{u}^{\prime}=S^{e}
\end{array}\right.
$$

Possible source terms $\left(S^{\rho}, \boldsymbol{S}^{m}, S^{e}\right)$ are also introduced in these equations to elaborate an hybrid acoustic approach in the next section. From the homogeneous system, the substitution of different variables for the benefit of the fluctuating pressure then leads to

$$
\mathcal{L}_{0}\left[p^{\prime}\right]=0 \quad \text { where } \quad \mathcal{L}_{0} \equiv \frac{\bar{D}}{\bar{D} t}\left[\frac{\bar{D}^{2}}{\bar{D} t^{2}}-\frac{\partial}{\partial x_{i}}\left(\bar{c}^{2} \frac{\partial}{\partial x_{i}}\right)\right]+2 \bar{c}^{2} \frac{d \bar{u}_{1}}{d x_{l}} \frac{\partial^{2}}{\partial x_{1} \partial x_{l}}
$$

By convention in what follows, the summation is performed over $l=2,3$ for the $l$ indice, that is for the transverse directions with respect to the mean velocity $\bar{u}_{1}$. Comparison between Eq. (23) and Eq. (25) shows that at leading-order, the wave operator of Lilley's equation is the correct wave operator containing all mean flow acoustic interactions $[25,38]$. Therefore, acoustic source terms can be clearly defined in this context. Historically, Eq. (25) was introduced by Pridmore-Brown [69] in a slightly different form obtained by taking the Fourier transform in homogeneous directions $t$ and $x_{1}$.

\subsection{An hybrid approach based on linearized Euler equations}

Since the linearized Euler equations (LEE) include all mean flow - acoustic interactions by construction, a natural approach then consists in forcing the homogeneous LEE system (52) with suitable source terms. The exact linear wave operator $\mathcal{L}_{0}$ can be derived for a sheared mean flow, and by comparison with Lilley's equation (23), it is clear that source terms can formally be identified. One of the first applications of this numerical strategy can be found in Béchara et al. [4] for predicting subsonic jet noise.

In what follows, expressions of source terms associated with aerodynamic and combustion noise are derived for LEE. Taking into account of source terms in system (52), the fluctuating pressure $p^{\prime}$ satisfies the following inhomogeneous wave equation,

$$
\mathcal{L}_{0}\left[p^{\prime}\right]=\frac{\bar{D}^{2} S^{e}}{\bar{D} t^{2}}-\bar{c}^{2} \frac{\bar{D}}{\bar{D} t}\left[\bar{\rho} \nabla \cdot\left(\frac{1}{\bar{\rho}} \boldsymbol{S}^{m}\right)\right]+2 \bar{c}^{2} \frac{\partial \bar{u}_{1}}{\partial x_{l}} \frac{\partial S_{l}^{m}}{\partial x_{1}}
$$


This equation can not directly be compared to Lilley's equation (23). In following the original study of Lilley [53], the starting point is again to consider Phillips' equation

$$
\frac{d^{2} \pi}{d t^{2}}-\frac{\partial}{\partial x_{i}}\left(c^{2} \frac{\partial \pi}{\partial x_{i}}\right)=\frac{\partial u_{i}}{\partial x_{j}} \frac{\partial u_{j}}{\partial x_{i}}+\Lambda_{\mathrm{Ph}}
$$

where the expression of $\Lambda_{\mathrm{Ph}}$ is explicitly given by Eq. (19). Therefore, by introducing the decomposition of variables around the sheared mean flow, and by noting that $\pi=\bar{\pi}$ $+\pi^{\prime}$ with $\bar{\pi}=0$ for the considered mean flow, one gets

$$
\frac{\bar{D}^{2} \pi^{\prime}}{\bar{D} t^{2}}-\frac{\partial}{\partial x_{i}}\left(\bar{c}^{2} \frac{\partial \pi^{\prime}}{\partial x_{i}}\right)-2 \frac{\partial \bar{u}_{1}}{\partial x_{l}} \frac{\partial u_{l}^{\prime}}{\partial x_{1}}=\frac{\partial u_{i}^{\prime}}{\partial x_{j}} \frac{\partial u_{j}^{\prime}}{\partial x_{i}}+\Lambda_{\mathrm{Ph}}+\Lambda_{1}
$$

No linearization is introduced in the previous expression and the term $\Lambda_{1}$ contains higher order terms in the perturbation variable $\pi^{\prime}$. Using Eq. (45), the third-order differential equation based on Lilley's wave operator $\mathcal{L}_{0}$ can then be formed to obtain,

$$
\frac{1}{\bar{\rho} \bar{c}^{2}} \mathcal{L}_{0}\left[p^{\prime}\right]=\frac{\bar{D}}{\bar{D} t}\left(\frac{\partial u_{i}^{\prime}}{\partial x_{j}} \frac{\partial u_{j}^{\prime}}{\partial x_{i}}\right)-2 \frac{\partial \bar{u}_{1}}{\partial x_{l}} \frac{\partial}{\partial x_{1}}\left(u_{j}^{\prime} \frac{\partial u_{l}^{\prime}}{\partial x_{j}}\right)+\frac{\bar{D} \Lambda_{\mathrm{Ph}}}{\bar{D} t}+2 \frac{\partial \bar{u}_{1}}{\partial x_{l}} \frac{\partial}{\partial x_{1}}\left(\frac{1}{\rho} \frac{\partial \tau_{l j}}{\partial x_{j}}\right)+\Lambda_{2}
$$

where $\Lambda_{2}$ includes all higher order terms in the perturbation variables [53]. In this expression, the two first source terms correspond to aerodynamic noise,

$$
\Lambda_{\mathrm{ae}} \simeq \bar{c}^{2} \frac{\bar{D}}{\bar{D} t}\left(\bar{\rho} \frac{\partial}{\partial x_{i}} \frac{\partial u_{i}^{\prime} u_{j}^{\prime}}{\partial x_{j}}\right)-2 \bar{c}^{2} \frac{\partial \bar{u}_{1}}{\partial x_{l}} \frac{\partial}{\partial x_{1}}\left(\bar{\rho} \frac{\partial u_{j}^{\prime} u_{l}^{\prime}}{\partial x_{l}}\right)
$$

and by identification with Eq. (26), one gets the expression of $\boldsymbol{S}^{m}$,

$$
S_{i}^{m} \equiv-\bar{\rho} \frac{\partial u_{i}^{\prime} u_{j}^{\prime}}{\partial x_{j}}
$$

The incompressibility condition $\nabla \cdot \boldsymbol{u}^{\prime}=0$ has been used to simplify the source term $\Lambda_{\mathrm{ae}}$ in expression (29), and thus to formally obtain an expression corresponding to a quadrupole source term. This is a reasonable assumption to interpret real source terms [38] associated with an exact wave operator. Colonius et al. [18] have shown that the noise radiated by a low-Mach-number mixing layer is accurately reproduced by solving $\mathcal{L}_{0}\left[p^{\prime}\right]=\Lambda_{\mathrm{ae}}$, with $\Lambda_{\mathrm{ae}}$ given by expression (29). Furthermore, Goldstein [37] has reformulated the linearized Euler equations by introducing a new variable $\tilde{\pi}=$ $\left(p^{\prime} / \bar{p}\right)^{1 / \gamma}-1$ in order to directly obtain the quadrupolar form of this source term. A 
complete discussion of Lilley's equation including the recent works of Goldstein [35-37] can be found in Musafir [58].

A similar reasoning for combustion noise leads to the following expression for $\Lambda_{\mathrm{co}}$,

$$
\Lambda_{\mathrm{co}} \simeq \bar{\rho} \bar{c}^{2} \frac{\bar{D}^{2}}{\bar{D} t^{2}}\left(\frac{\gamma-1}{\rho c^{2}} \dot{\omega}_{T}\right)
$$

by only retaining the main contribution and neglecting all molecular transport terms and variations of the molar composition. By identification, the monopole source term in (52) therefore takes the following form,

$$
S^{e}=\bar{\rho} \bar{c}^{2} \frac{\gamma-1}{\rho c^{2}} \dot{\omega}_{T}
$$

Finally, LEE system (52) associated with source terms (30) and (32) defines an acoustic-analogy approach, which exactly corresponds to Lilley's equation at leading order for a sheared mean flow, i.e. the fluctuating pressure satisfies the wave equation $\mathcal{L}_{0}\left[p^{\prime}\right]=\Lambda_{\text {ae }}+\Lambda_{\text {co. }}$

The linearized Euler equations do not only describe acoustic perturbations, because they can also be interpreted as a generalization of Rayleigh's equation for compressible flows $[3,25,38]$. As a result, instability waves of the Kelvin-Helmholtz type [1] are also solutions of LEE. These solutions can be removed by solving the linearized Euler equations in the frequency domain, as shown by Rao \& Morris [71] or by Karabasov et al. [47]. Another way to tackle this problem is to simplify LEE system using high-frequency assumptions, as proposed by Bogey et al. [10] in calculations of noise generated by vortex pairings in a mixing layer, see also the discussion by Bailly \& Bogey [3] not reproduced here.

\subsection{Acoustic perturbation equations}

Bui et al. [11] propose to use the acoustic perturbation equations (APE) system combined with source tepms in order to investigate combustion noise. The homogeneous APE system is used by the authors [29] to describe wave propagation in the presence of a mean flow without exciting vortical or entropy waves, and the source terms are derived from the conservation equations for reacting flows. The set of equations (APE-2 variant) to solve is

$$
\left\{\begin{array}{l}
\partial_{t} \rho^{\prime}+\partial_{x_{i}}\left(\rho^{\prime} \bar{u}_{i}+\bar{\rho} u_{i}^{\prime}\right)=q_{c} \\
\partial_{t} u_{i}^{\prime}+\partial_{x_{j}}\left(\bar{u}_{i} u_{j}^{\prime}\right)+\partial_{i}\left(p^{\prime} / \bar{\rho}\right)=\boldsymbol{q}_{m} \\
\partial_{t} p^{\prime}-\bar{c}^{2} \partial_{t} \rho^{\prime}=q_{e}
\end{array}\right.
$$

and the complete expression of the source terms may be found in Bui et al. [11]. As illustration, the dominant source term in $\boldsymbol{q}_{m}$ used for predicting aerodynamic noise is 
based on the Lamb vector, namely $\boldsymbol{q}_{m} \simeq-(\boldsymbol{\omega} \times \boldsymbol{u})^{\prime}$. The homogeneous system (33) describes sound propagation in an irrotational mean flow, and the APE may be interpreted as an extension of the theory of vortex sound $[29,42,55]$. A stable system is then obtained for hydrodynamic perturbations under this assumption, as discussed in Möhring [55] for instance. All refraction effects are however not included in the homogeneous system (33), as documented numerically by results of the third computational aeroacoustics workshop $[1,60]$ or theoretically by comparison with LEE. As in Lighthill's analogy, an exact solution of the problem could be retrieved by using equivalent source terms on the right-hand side of the APE system, but acoustic interactions are then included in these source terms and should be computed in advance.

For isobaric combustion in the low Mach number limit, Bui et al. [11] have retained a simplified formulation of their APE-RF source terms, given by

$$
q_{e} \simeq-\bar{c}^{2} \frac{\bar{\rho}}{\rho} \frac{d \rho}{d t}
$$

whereas $q_{c} \simeq 0$ and $\boldsymbol{q}_{m} \simeq 0$. This source term is directly computed from an incompressible LES simulation of turbulent reacting flow, and good results are reported by comparison with experimental data regarding a turbulent non-preximed flame. It seems interesting to note that $q_{e}$ is directly linked to the heat release through Eq. (42), that is $q_{e}=(\gamma-1) \dot{\omega}_{T}=S_{e}$, under the same assumptions.

\section{CONCLUDING REMARKS}

This article considers the derivation of inhomogeneous wave equations which can be applied to the analysis of combustion noise from reacting flows. This is used to identify noise sources, and formulate a suitable hybrid approach combining a wave operator and source terms. A specific point in combustion is the presence of strong temperature and pressure gradients, which generate strong interactions with the fluctuating fields and produce additional noise. This indirect combustion noise usually dominates core noise in aircraft engines. Several numerical studies have already been performed in this framework, and the developments of computational aeroacoustics should continue to support these advances in the future. Finally, the relative importance of the different source terms can be estimated by comparison with direct noise computation. This has been successfully applied to aerodynamic noise and should also be done carefully for combustion noise.

\section{ACKNOWLEDGMENTS}

The authors are pleased to contribute to this special issue of the International Journal of Aeroacoustics in honor of Geoffrey Lilley. Geoffrey has visited the Centre Acoustique of Ecole Centrale de Lyon several times, participing as referee for several 
Ph.D. defenses and as seminar speaker. The authors are also grateful to Geoffrey for helpul discussions about turbulence, jet noise and large-eddy simulations.

The work has been partially supported by the Fondation de Recherche pour 1 'Aéronautique \& 1'Espace (FRAE - BRUCO Project). The first and the third authors are also with Institut Universitaire de France.

\section{A. DERIVATION OF AN ALTERNATIVE EXPRESSION FOR THE ENERGY CONSERVATION}

The energy balance (3) can be written in the following form [68]

$$
\rho \frac{d h}{d t}=\frac{d p}{d t}-\nabla \cdot \boldsymbol{q}+\boldsymbol{\tau}: \nabla \boldsymbol{u}
$$

by introducing the enthalpy of the mixture $h$. This enthalpy variable is defined as the sum of two contributions, associated with temperature changes and with the formation of species respectively,

$$
h=h_{s}+\sum_{k} \Delta h_{f, k}^{0} Y_{k}=\sum_{k} h_{s, k} Y_{k}+\sum_{k} \Delta h_{f, k}^{0} Y_{k}
$$

where $h_{s}$ is the sensible enthalpy of the mixture, $\Delta h_{f, k}^{0}$ is the enthalpy of formation of species $k, h_{s, k}$ the sensible enthalpy of species $k$. Furthermore, $d h_{s, k}=c_{p, k} d T$ where $c_{p, k}$ is the specific heat of species $k$ at constant pressure and $T$ the temperature. In Eq. (34), the heat flux $q$ is the sum of the Fourier law and an additional term taking into account heat transfer by species diffusion, namely

$$
\boldsymbol{q}=-\lambda \nabla T+\rho \sum_{k} h_{k} Y_{k} \boldsymbol{v}_{k}
$$

where $\lambda$ is the thermal diffusivity, $h_{k}=h_{s, k}+\Delta h_{f, k}^{0}$ and $\boldsymbol{v}_{k}$ is the diffusion velocity of species $k$. Details about all theses quantities can be found in Poinsot \& Veynante [68] for instance.

Equation (34) is the starting point for the following developments, with the aim of deriving a relation for the term $d p / d t-c^{2} d \rho / d t$. First, $d h / d t$ is developed using the definition of enthalpy, yielding

$$
\begin{aligned}
\frac{d h}{d t} & =\frac{d h_{s}}{d t}+\sum_{k} \Delta h_{f, k}^{0} \frac{d Y_{k}}{d t}=\sum_{k} h_{s, k} \frac{d Y_{k}}{d t}+\sum_{k} c_{p, k} Y_{k} \frac{d T}{d t}+\sum_{k} \Delta h_{f, k}^{0} \frac{d Y_{k}}{d t} \\
& =\sum_{k} h_{s, k} \frac{d Y_{k}}{d t}+c_{p} \frac{d T}{d t}+\sum_{k} \Delta h_{f, k}^{0} \frac{d Y_{k}}{d t}
\end{aligned}
$$


By noting that the temperature is a function of $T=T\left(\rho, p, Y_{k}\right)$, its exact differential is given by

$$
\begin{aligned}
d T & =\left.\frac{\partial T}{\partial p}\right|_{\rho, Y_{k}} d p+\left.\frac{\partial T}{\partial \rho}\right|_{p, Y_{k}} d \rho+\left.\sum_{k} \frac{\partial T}{\partial Y_{k}}\right|_{\rho, p, Y_{l \neq k}} d Y_{k} \\
& =\frac{1}{\rho r} d p-\frac{T}{\rho} d \rho-T \sum_{k} \frac{W}{W_{k}} d Y_{k}
\end{aligned}
$$

for a perfect gas in which $W_{k}$ is the molar weight of species $k$ and the following relation $r=R / W=R \Sigma_{k} Y_{k} / W_{k}$ has been also used for simplifications. The last term in Eq. (37) may be rearranged in a simple way. Indeed, it follows from the definition of the gas constant $r$ that

$$
\frac{d}{d t}(\ln r)=\frac{1}{r} \frac{d r}{d t}=\frac{1}{r} \frac{d}{d t}\left(R \sum_{k} \frac{Y_{k}}{W_{k}}\right)=\frac{W}{W_{k}} \frac{d Y_{k}}{d t}
$$

Therefore, by substituting Eq. (37) into Eq. (34), and by using the two relations (37) and (38), one gets

$$
\frac{d \rho}{d t}=\frac{1}{c^{2}} \frac{d p}{d t}+\frac{1}{c_{p} T}[\nabla \cdot \boldsymbol{q}-\boldsymbol{\tau}: \nabla \boldsymbol{u}+\underbrace{}_{k} \sum_{k, k}\left(h_{s, k}+\Delta h_{f, k}^{0}\right) \frac{d Y_{k}}{d t}]-\rho \frac{d}{d t}(\ln r)
$$

By introducing the heat release per unit volume $\dot{\omega}_{T}$ due to combustion from Eq. (4), the underlined term denoted (a) hereafter reduces to

$$
\underline{\text { (a) }}=\sum_{k}\left(h_{s, k}+\Delta h_{f, k}^{0}\right)\left(\dot{\omega}_{k}-\nabla \cdot \boldsymbol{J}_{k}\right)=-\dot{\omega}_{T}-\sum_{k} h_{k} \nabla \cdot \boldsymbol{J}_{k}
$$

Furthermore, the molecular diffusion terms may be written as follows

$$
\begin{aligned}
\nabla \cdot \boldsymbol{q}-\sum_{k} h_{k} \nabla \cdot \boldsymbol{J}_{k} & =\nabla \cdot\left(-\lambda \nabla T+\rho \sum_{k} h_{k} Y_{k} \boldsymbol{v}_{k}\right)-\sum_{k} h_{k} \nabla \cdot\left(\rho Y_{k} \boldsymbol{v}_{k}\right) \\
& =-\nabla \cdot(\lambda \nabla T)+\rho \sum_{k} Y_{k} c_{p, k} \boldsymbol{v}_{k} \cdot \nabla T
\end{aligned}
$$

Finally, combining Eq. (39), Eq. (40) and Eq. (41) leads to

$$
\begin{aligned}
\frac{d \rho}{d t}= & \frac{1}{c^{2}} \frac{d p}{d t}+\frac{\gamma-1}{c^{2}}\left[-\dot{\omega}_{T}-\nabla \cdot(\lambda \nabla T)+\rho \sum_{k} Y_{k} c_{p, k} \boldsymbol{v}_{k} \cdot \nabla T-\boldsymbol{\tau}: \nabla \boldsymbol{u}\right] \\
& -\rho \frac{d}{d t}(\ln r)
\end{aligned}
$$


The conservation of energy may thus be expressed as Eq. (3), or as Eq. (34), or equivalently $[12,68]$ as Eq. (42). Note that Eq. (42) is denoted by Eq. (7) in the main text to simplify reading. The well known isentropic relation $d p / d t=c^{2} d \rho / d t$ is found for an ideal perfect gas. It should also be noted in passing that the definition of the heat release used in this work, [68] is $\dot{\omega}_{T}=-\Sigma_{k}\left(h_{s, k}+\Delta h_{f, k}^{0}\right) \dot{\omega}_{k}$ The term $\rho \Sigma_{k} Y_{k} c_{p, k} \boldsymbol{v}_{k} \cdot \nabla T$ is associated with diffusion of species with different enthalpies whereas the last term $\rho d(\ln r) / d t$ is associated with the change of the mean molar weight of the mixture during combustion, see also Candel et al. [12] for a more detailed discussion. Simplified formulations of Eq. (42) can be found in the litterature $[14,15,19,48,83]$ under different approximations including isobaric, isomolar or low Mach number reacting flow assumptions.

\section{B. PHILLIPS' EOUATION FOR A NON-REACTING FLOW}

As a starting point, one considers the balance of mass (1), momentum (2) and energy for a perfect gas written as

$$
\frac{d p}{p}-\gamma \frac{d \rho}{\rho}=\frac{1}{c_{v}} d s
$$

where $s$ represents the entropy of the fluid. From the balance of mass, it follows that

$$
\frac{\partial u_{j}}{\partial x_{j}}=-\frac{1}{\rho} \frac{d \rho}{d t}=-\frac{1}{\gamma p} \frac{d p}{d t}+\frac{1}{c_{p}} \frac{d s}{d t}
$$

A new variable $\pi$ based on the logarithm of the pressure is introduced by Phillips ${ }^{64}$

$$
\pi=\frac{1}{\gamma} \ln \left(\frac{p}{p_{\infty}}\right)
$$

which allows to reformulate the set of equations as

$$
\begin{gathered}
\frac{d \pi}{d t}+\frac{\partial u_{j}}{\partial x_{j}}-\frac{1}{c_{p}} \frac{d s}{d t}=0 \\
\frac{d u_{i}}{d t}+c^{2} \frac{\partial \pi}{\partial x_{i}}-\frac{1}{\rho} \frac{\partial \tau_{i j}}{\partial x_{j}}=0
\end{gathered}
$$

A wave equation can then be obtained by considering the arrangement $d(44) / d t$ $-\nabla \cdot(45)=0$ in order to remove the linear velocity term, which yields

$$
\frac{d}{d t}\left[\frac{d \pi}{d t}+\frac{\partial u_{j}}{\partial x_{j}}-\frac{1}{c_{p}} \frac{d s}{d t}\right]-\frac{\partial}{\partial x_{i}}\left[\frac{d u_{i}}{d t}+c^{2} \frac{\partial \pi}{\partial x_{i}}-\frac{1}{\rho} \frac{\partial \tau_{i j}}{\partial x_{j}}\right]=0
$$


Noting that the partial derivative $\partial_{x_{i}}$ does not commute with the material derivative $d / d t$, that is

$$
\frac{\partial}{\partial x_{i}} \frac{d}{d t}=\frac{d}{d t} \frac{\partial}{\partial x_{i}}+\frac{\partial u_{j}}{\partial x_{i}} \frac{\partial}{\partial x_{j}}
$$

the convected wave equation of Phillips [64] is finally obtained

$$
\frac{d^{2} \pi}{d t^{2}}-\frac{\partial}{\partial x_{i}}\left(c^{2} \frac{\partial \pi}{\partial x_{i}}\right)=\frac{\partial u_{i}}{\partial x_{j}} \frac{\partial u_{j}}{\partial x_{i}}+\frac{d}{d t}\left(\frac{1}{c_{p}} \frac{d s}{d t}\right)-\frac{\partial}{\partial x_{i}}\left(\frac{1}{\rho} \frac{\partial \tau_{i j}}{\partial x_{j}}\right)
$$

This equation is discussed in Section 4.

\section{LILLEY' EOUATION FOR A NON-REACTING FLOW}

As shown in section 4, all the mean flow effects on sound propagation are not included in Eq. (46). For a sheared mean flow, the compressible linear part of the source term $\nabla \boldsymbol{u}: \nabla \boldsymbol{u}$ is indeed associated with refraction effects. The first inhomogeneous wave equation describing sound propagation in a shear flow with consistent source terms on the right-hand side was established by Lilley. [53] Following the derivation based on the linearized Euler equations, the material derivative is again applied to Eq. (46), to obtain

$$
\frac{d}{d t}\left[\frac{d^{2} \pi}{d t^{2}}-\frac{\partial}{\partial x_{i}}\left(c^{2} \frac{\partial \pi}{\partial x_{i}}\right)\right]=2 \frac{\partial u_{i}}{\partial x_{j}} \frac{d}{d t}\left(\frac{\partial u_{j}}{\partial x_{i}}\right)+\frac{d^{2}}{d t^{2}}\left(\frac{1}{c_{p}} \frac{d s}{d t}\right)-\frac{d}{d t}\left[\frac{\partial}{\partial x_{i}}\left(\frac{1}{\rho} \frac{\partial \tau_{i j}}{\partial x_{j}}\right)\right]
$$

The first term on the right-hand side can then be developed as follows,

$$
\begin{aligned}
2 \frac{\partial u_{i}}{\partial x_{j}} \frac{d}{d t}\left(\frac{\partial u_{j}}{\partial x_{i}}\right) & =2 \frac{\partial u_{i}}{\partial x_{j}} \frac{\partial}{\partial x_{i}}\left(\frac{d u_{j}}{d t}\right)-2 \frac{\partial u_{i}}{\partial x_{j}} \frac{\partial u_{j}}{\partial x_{k}} \frac{\partial u_{k}}{\partial x_{i}} \\
& =-2 \frac{\partial u_{i}}{\partial x_{j}} \frac{\partial}{\partial x_{i}}\left(c^{2} \frac{\partial \pi}{\partial x_{j}}\right)+2 \frac{\partial u_{i}}{\partial x_{j}} \frac{\partial}{\partial x_{i}}\left(\frac{1}{\rho} \frac{\partial \tau_{i j}}{\partial x_{i}}\right)-2 \frac{\partial u_{i}}{\partial x_{j}} \frac{\partial u_{j}}{\partial x_{k}} \frac{\partial u_{k}}{\partial x_{i}}
\end{aligned}
$$

by using Eq. (44) of the balance of momentum. Finally, another exact reformulation of the Navier-Stokes equations can be achieved in the form

$$
\begin{aligned}
\frac{d}{d t}\left[\frac{d^{2} \pi}{d t^{2}}\right. & \left.-\frac{\partial}{\partial x_{i}}\left(c^{2} \frac{\partial \pi}{\partial x_{i}}\right)\right]+2 \frac{\partial u_{i}}{\partial x_{j}} \frac{\partial}{\partial x_{i}}\left(c^{2} \frac{\partial \pi}{\partial x_{j}}\right)=-2 \frac{\partial u_{i}}{\partial x_{j}} \frac{\partial u_{j}}{\partial x_{k}} \frac{\partial u_{k}}{\partial x_{i}} \\
+ & 2 \frac{\partial u_{i}}{\partial x_{j}} \frac{\partial}{\partial x_{i}}\left(\frac{1}{\rho} \frac{\partial \tau_{i j}}{\partial x_{i}}\right)+\frac{d^{2}}{d t^{2}}\left(\frac{1}{c_{p}} \frac{d s}{d t}\right)-\frac{d}{d t}\left[\frac{\partial}{\partial x_{i}}\left(\frac{1}{\rho} \frac{\partial \tau_{i j}}{\partial x_{j}}\right)\right]
\end{aligned}
$$

as originally proposed by Lilley [53] (1972), and reformulated by Doak [25,58]. 


\section{THE LINEARIZED EULER EQUATIONS}

The Euler equations linearized around an arbitrarily steady flow govern sound propagation in this flow $[7,38,66]$. A non-conservative form of these linearized Euler Equations is

$$
\begin{gathered}
\partial_{t} \rho^{\prime}+\overline{\boldsymbol{u}} \cdot \nabla \rho^{\prime}+\boldsymbol{u}^{\prime} \cdot \nabla \bar{\rho}+\bar{\rho} \nabla \cdot \boldsymbol{u}^{\prime}+\rho^{\prime} \nabla \cdot \overline{\boldsymbol{u}}=0 \\
\partial_{t}\left(\bar{\rho} \boldsymbol{u}^{\prime}\right)+\nabla \cdot\left(\bar{\rho} \boldsymbol{u}^{\prime} \overline{\boldsymbol{u}}\right)+\left(\rho^{\prime} \overline{\boldsymbol{u}}+\bar{\rho} \boldsymbol{u}^{\prime}\right) \cdot \nabla \overline{\boldsymbol{u}}+\nabla p^{\prime}=0 \\
\partial_{t} p^{\prime}+\nabla \cdot\left(p^{\prime} \overline{\boldsymbol{u}}+\gamma \bar{p} \boldsymbol{u}^{\prime}\right)+(\gamma-1) p^{\prime} \nabla \cdot \overline{\boldsymbol{u}}-(\gamma-1) \boldsymbol{u}^{\prime} \cdot \nabla \bar{p}=0
\end{gathered}
$$

for an ideal perfect gas, where $\rho=\bar{\rho}+\rho^{\prime}, \boldsymbol{u}=\overline{\boldsymbol{u}}+\boldsymbol{u}^{\prime}$ and $p=\bar{p}+p^{\prime}$ and by using the following convention, $\left.\nabla \cdot\left(\bar{\rho} \boldsymbol{u}^{\prime} \overline{\boldsymbol{u}}\right)\right|_{i}=\partial_{x_{j}}\left(\bar{\rho} u_{i}^{\prime} \bar{u}_{j}\right)$. For the present study, it is more convenient to work with the pressure fluctuation. Furthermore, from Eq. (50) and Eq. (48), and by noting that $\gamma \bar{p}=\bar{\rho} \bar{c}^{2}$,

$$
\begin{aligned}
\partial_{t} p^{\prime}+\overline{\boldsymbol{u}} \cdot \nabla p^{\prime}+\boldsymbol{u}^{\prime} \cdot \nabla \bar{p} & =-\gamma \bar{p} \nabla \cdot \boldsymbol{u}^{\prime}-\gamma p^{\prime} \nabla \cdot \overline{\boldsymbol{u}} \\
& =\bar{c}^{2}\left(\partial_{t} \rho^{\prime}+\overline{\boldsymbol{u}} \cdot \nabla \rho^{\prime}+\boldsymbol{u}^{\prime} \cdot \nabla \bar{\rho}+\rho^{\prime} \nabla \cdot \overline{\boldsymbol{u}}\right)-\gamma p^{\prime} \nabla \cdot \overline{\boldsymbol{u}} \\
& =\bar{c}^{2}\left(\partial_{t} \rho^{\prime}+\overline{\boldsymbol{u}} \cdot \nabla \rho^{\prime}+\boldsymbol{u}^{\prime} \cdot \nabla \bar{\rho}\right)+\left(\bar{c}^{2} \rho^{\prime}-\gamma p^{\prime}\right) \nabla \cdot \overline{\boldsymbol{u}} \\
& =\bar{c}^{2}\left(\partial_{t} \rho^{\prime}+\overline{\boldsymbol{u}} \cdot \nabla \rho^{\prime}+\boldsymbol{u}^{\prime} \cdot \nabla \bar{\rho}\right)+\bar{c}^{2}{ }^{\prime} \overline{\boldsymbol{u}} \cdot \nabla \overline{\boldsymbol{u}}
\end{aligned}
$$

where $\bar{c}^{2} \simeq \bar{c}^{2}\left(p^{\prime} / \bar{p}-\rho^{\prime} / \bar{\rho}\right)$. This relation can also be obtained by linearization of $d p / d t$ $=c^{2} d \rho / d t$.

From these equations (48)-(51), an homogeneous wave equation can be derived for a stratified mean flow, that is $\bar{u}_{i}=\bar{u}_{1}\left(x_{2}, x_{3}\right) \delta_{1 i}$ for the mean velocity, whereas $\bar{\rho}=\bar{\rho}\left(x_{2}, x_{3}\right)$ and $\bar{c}=\bar{c}\left(x_{2}, x_{3}\right)$ are only functions of transverse coordinates, the mean pressure then being constant. Noting $\bar{D}_{t} \equiv \partial_{t}+\bar{u}_{1} \partial_{x_{1}}$ the material derivative along the mean flow, system (48)-(50) then reduces to

$$
\begin{gathered}
\bar{D}_{t} \rho^{\prime}+\nabla \cdot\left(\bar{\rho} \boldsymbol{u}^{\prime}\right)=0 \\
\bar{D}_{t}\left(\bar{\rho} \boldsymbol{u}^{\prime}\right)+\bar{\rho} \boldsymbol{u}^{\prime} \cdot \nabla \overline{\boldsymbol{u}}+\nabla p^{\prime}=\boldsymbol{S}^{m} \\
\bar{D}_{t} p^{\prime}+\gamma \bar{p} \nabla \cdot \boldsymbol{u}^{\prime}=S^{e}
\end{gathered}
$$

Possible source terms $\left(S^{\rho}, \boldsymbol{S}^{m}, S^{e}\right)$ are also introduced in these equations, in connection with section 5.2 to elaborate an acoutic analogy based on linearized Euler's Equations. Then, by taking the usual combination $\bar{D}_{t}(52)-\nabla \cdot(53)$, it follows that

$$
\bar{D}_{t}^{2} \rho^{\prime}+\bar{D}_{t} \nabla \cdot\left(\bar{\rho} \boldsymbol{u}^{\prime}\right)-\nabla \cdot \bar{D}_{t}\left(\bar{\rho} \boldsymbol{u}^{\prime}\right)-\nabla \cdot\left(\bar{\rho} \boldsymbol{u}^{\prime} \cdot \nabla \overline{\boldsymbol{u}}\right)-\nabla^{2} p^{\prime}=\bar{D}_{t} S_{\rho}-\nabla \cdot \boldsymbol{S}^{m}
$$


which can be simplified by noting that for the considered sheared mean flow field,

$$
\bar{D}_{t} \nabla \cdot\left(\bar{\rho} \boldsymbol{u}^{\prime}\right)-\nabla \cdot \bar{D}\left(\bar{\rho} \boldsymbol{u}^{\prime}\right)-\nabla \cdot\left(\bar{\rho} \boldsymbol{u}^{\prime} \cdot \nabla \overline{\boldsymbol{u}}\right)=-2 \partial_{x_{l}} \bar{u}_{1} \partial_{x_{1}}\left(\bar{\rho} u_{l}^{\prime}\right)
$$

where summation over indice $l$ is performed for the transverse directions $l=2,3$. Furthemore, the combination of Eq. (54) and (52) leads to

$$
\bar{D}_{t}^{2} \rho^{\prime}=\left(1 / \bar{c}^{2}\right) \bar{D}_{t}^{2} p^{\prime}-\bar{D}_{t} u_{l}^{\prime} \nabla_{x_{l}} \bar{\rho}+\bar{D}_{t}\left(S^{\rho}-S^{e} / \bar{c}^{2}\right)
$$

after rearranging the different terms. The convected wave equation (55) can then be written as

$$
\left(1 / \bar{c}^{2}\right) \bar{D}_{t}^{2} p^{\prime}-\bar{D}_{t} u_{l}^{\prime} \nabla_{x_{l}} \bar{\rho}-2 \partial_{x_{l}} \bar{u}_{1} \partial_{x_{1}}\left(\bar{\rho} u_{l}^{\prime}\right)-\nabla^{2} p^{\prime}=\bar{D}_{t}\left(S^{e} / \bar{c}^{2}\right)-\nabla \cdot \boldsymbol{S}^{m}
$$

In addition, considering Eq. (53) for the transverse velocity component $l$, and multiplying each side by the mean density gradient, it follows that

$$
-\bar{D}_{t} u_{l}^{\prime} \nabla_{x_{l}} \bar{\rho}=(1 / \bar{\rho}) \nabla p^{\prime} \cdot \nabla \bar{\rho}-(1 / \bar{\rho}) \boldsymbol{S}^{m} \cdot \nabla \bar{\rho}
$$

which enables us to write the convected wave equation (57) as

$$
\begin{aligned}
\left(1 / \bar{c}^{2}\right) \bar{D}_{t}^{2} p^{\prime}-2 \partial_{x_{l}} \bar{u}_{1} \partial_{x_{1}}\left(\bar{\rho} u_{l}^{\prime}\right)+(1 / \bar{\rho}) \nabla p^{\prime} \cdot \nabla \bar{\rho}-\nabla^{2} p^{\prime}= & \bar{D}_{t}\left(S^{e} / \bar{c}^{2}\right)-\nabla \cdot \boldsymbol{S}^{m} \\
& +(1 / \bar{\rho}) \boldsymbol{S}^{m} \cdot \nabla \bar{\rho}
\end{aligned}
$$

Then, using the two identities,

$$
\begin{aligned}
& \bar{c}^{2}\left[(1 / \bar{\rho}) \nabla p^{\prime} \cdot \nabla \bar{\rho}-\nabla^{2} p^{\prime}\right]=-\nabla \cdot\left(\bar{c}^{2} \nabla p^{\prime}\right) \\
& -\nabla \cdot \boldsymbol{S}^{m}+(1 / \bar{\rho}) \boldsymbol{S}^{m} \cdot \nabla \bar{\rho}=-\bar{\rho} \nabla \cdot\left(\boldsymbol{S}^{m} / \bar{\rho}\right)
\end{aligned}
$$

and furthermore, by noting that

$$
\bar{D}_{t} \partial_{x_{l}} \bar{u}_{1} \partial_{x_{1}}\left(\bar{\rho} u_{l}^{\prime}\right)=\partial_{x_{l}} \bar{u}_{1} \partial_{x_{1}} \bar{D}_{t}\left(\bar{\rho} u_{l}^{\prime}\right)=\partial_{x_{l}} \bar{u}_{1} \partial_{x_{1}}\left[-\partial_{x_{l}} p^{\prime}+S_{l}^{m}\right]
$$

the following inhomogeneous wave equation is finally obtained by applying the material derivative operator $\bar{D}_{t}$ to Eq. (57),

$$
\begin{gathered}
\bar{D}_{t}\left\{\left(1 / \bar{c}^{2}\right) \bar{D}_{t}^{2} p^{\prime}-\bar{\rho} \nabla \cdot\left[(1 / \bar{\rho}) \nabla p^{\prime}\right]\right\}+2 \partial_{x_{l}} \bar{u}_{1} \partial_{x_{1} x_{l}}^{2} p^{\prime} \\
=\bar{D}_{t}\left[\bar{D}_{t}\left(S^{e} / \bar{c}^{2}\right)-\bar{\rho} \nabla \cdot\left(\boldsymbol{S}^{m} / \bar{\rho}\right)\right]+2 \partial_{x_{l}} \bar{u}_{1} \partial_{x_{1}} S_{l}^{m}
\end{gathered}
$$

This wave equation, derived from linearized Euler's Equations by assuming a stratified mean flow field, is discussed in sections 5.1 and 5.2. 


\section{REFERENCES}

[1] Agarwal, A., Morris, P. J. \& Mani, R., 2004, Calculation of sound propagation in nonuniform flows: suppression of instability waves, AIAA Journal, 42(1), 80-88.

[2] Bailly, C., Bogey, C. and Gloerfelt, X., 2005, Some useful hybrid approaches for predicting aerodynamic noise, C. R. Me., Acad. Sci. Paris, 333(9), 666-675.

[3] Bailly, C. \& Bogey, C., 2004, Contributions of computational aeroacoustics to jet noise research and prediction, International Journal of Computational Fluid Dynamics, 18(6), 481-491.

[4] Béchara, W., Bailly, C., Lafon, P. \& Candel, S., 1994, Stochastic approach to noise modeling for free turbulent flows, AIAA Journal, 32(3), 455-463.

[5] Becker, S., Hahn, C., Kaltenbacher, M. \& Lerch, R., 2008, Flow-induced sound of wall-mounted cylinders with different geometries, AIAA Journal, 46(9), 2265-2281.

[6] Blacodon, D., 2009, Combustion-noise characterization of a turbofan engine with a spectral estimation method, Journal Prop. Power, 25(2), 374-379.

[7] Blokhintzev D., 1946, The propagation of sound in an inhomogeneous and moving medium I, J. Acoust. Soc. Am., 18(2), 322-328.

[8] Bogey, C. \& Bailly, C. 2007, An analysis of the correlations between the turbulent flow and the sound pressure field of turbulent jets, J. Fluid Mech., 583, 71-97.

[9] Bogey, C., Gloerfelt, X. \& Bailly, C., 2003, An illustration of the inclusion of sound-flow interactions in Lighthill's equation, AIAA Journal, 41(8), 1604-1606.

[10] Bogey, C., Bailly, C. \& Juvé, D., 2002, Computation of flow noise using source terms in linearized Euler's equations, AIAA Journal, 40(2), 235-243.

[11] Bui, T. P., Schröder, W. \& Meinke, M., 2007, Acoustic perturbation equations for reacting flows to compute combustion noise, International Journal of Aeroacoustics, 6(4), 335-355.

[12] Candel, S., Durox, S., Ducruix, S., Birbaud, A.-L., Noiray, N. \& Schuller, T., 2009, Flame dynamics and combustion noise: progress and challenges, International Journal of Aeroacoustics, 8(1 \& 2),1-56.

[13] Candel, S. M., 1972, Analytical studies of some acoustic problems of jet engines, Ph.D. Thesis, California Institute of Technology.

[14] Chiu, H. H. \& Summerfield, M., 1974, Theory of combustion noise, Acta Astronautica, 1, 967-984.

[15] Clavin, P., 1994, Premixed combustion and gasdynamics, Annu. Rev. Fluid Mech., 26, 321-352.

[16] Clavin, P. \& Siggia, E., 1991, Turbulent premixed flames and sound generation, Combust. Sci. and Tech., 78, 147-155.

[17] Colonius, T. and Lele, S., 2004, Computational aeroacoustics: progress on nonlinear problems on sound generation, Progress in Aerospace Sciences, 40, 345-416.

[18] Colonius, T., Lele, S. K. \& Moin, P., 1997, Sound generation in a mixing layer, J. Fluid Mech., 330, 375-409. 
[19] Crighton, D. G., Dowling, A. P., Ffowcs Williams, J. E., Heckl, M. \& Leppington, F. G., 1992, Modern methods in analytical acoustics, Springer-Verlag, London.

[20] Crighton, D., 1975, Basic principles of aerodynamic noise generation, Progress in Aerospace Sciences, 16(1), 31-96.

[21] Culick, F., 2006, Unsteady Motions in Combustion Chambers for Propulsion Systems, AGARD, RTO-AG-AVT-039.

[22] Cumpsty, N.A. \& Marble, F.E., 1977, Core noise gas turbine exhausts, J. Sound Vib., 54(2), 297-309.

[23] Cumpsty, N.A. \& Marble, F.E., 1977, The interaction of entropy fluctuations with turbine blade rows; a mechanism of turbujet engine noise, Proc. R. Soc. Lond. A, 357, 323-344.

[24] Cumpsty, N.A., 1979, Jet engine combustion noise: pressure, entropy and vorticity perturbations produced by unsteady combustion or heat addition, J. Sound Vib., 66(4), 527-544.

[25] Doak, P. E., 1972, Analysis of internally generated sound in continuous materials: 2. A critical review of the conceptual adequacy and physical scope of existing theories of aerodynamic noise, with special reference to supersonic jet noise, J. Sound Vib., 25(2), 263-335.

[26] Dowling, A.P. \& Morgans, A.S., 2005, Feedback control of combustion oscillations, Annu. Rev. Fluid Mech., 37, 151-182.

[27] Ducruix, S., Schuller, T., Durox, D. \& Candel, S., 2003, Combustion dynamics and instabilities: elementary coupling and driving mechanisms, Journal Prop. Power, 19(5), 722-734.

[28] Durox, S., Schuller, T., Noiray, N., Birbaud, A. L. \& Candel, S., 2009, Rayleigh criterion and acoustic energy balance in unconfined self-sustained oscillating flames, Combustion and Flame, 156, 106-119.

[29] Ewert, R. \& Schroder, W., 2003, Acoustic perturbation equations based on flow decomposition via source filtering, J. Comput. Phys., 188, 365-398.

[30] Feldman Jr., K. T., 1968, Review of the litterature on Rijke thermoacoustic phenomena, J. Sound Vib., 7(1), 83-89.

[31] Ffowcs Williams, J. E., 1982, Sound sources in aerodynamics - Fact and fiction, AIAA Journal, 20(3), 307-315.

[32] Ffowcs Williams, J. E. \& Howe, M. S., 1975, The generation of sound by density inhomogeneities in low Mach number nozzle flows, J. Fluid Mech., 70(3), 605-622.

[33] Ffowcs Williams, J.E., 1968, Jet noise at very low and very high speed, AFOSRUTIAS Symposium on Aerodynamic Noise, Toronto, 131-145.

[34] Ffowcs Williams, J.E. \& Gordon, C. G., 1965, Noise of highly turbulent jets at low exhaust speeds, AIAA Journal, 3(4), 791-793.

[35] Goldstein, M.E., 2003, A generalized acoustic analogy, J. Fluid Mech., 488, 315-333.

[36] Goldstein, M.E., 1984, Aeroacoustics of turbulent shear flows, Annu. Rev. Fluid Mech., 16, 263-285. 
[37] Goldstein, M.E., 2001, An exact form of Lilley's equation with a velocity quadrupole/temperature dipole source term, J. Fluid Mech., 443, 231-236.

[38] Goldstein, M. E., 1976, Aeroacoustics, McGraw-Hill, New York.

[39] Harper-Bourne, M., Moore, A. \& Siller, H., 2008, A study of large aero-engine combustor noise, AIAA Paper 2008-2942.

[40] Hassan, H. A., 1974, Scaling of combustion-generated noise, J. Fluid Mech., 66(3), 445-453.

[41] Hoch, R. G., Thomas, P. \& Weiss, E., 1976, An experimental investigation of the core engine noise of a turbofan engine, Progress in Astronautics and Aeronautics, 43, 555-578.

[42] Howe, M.S., 1998, Acoustics of fluid-structure interactions, Cambridge University Press, Cambridge.

[43] Hubert, J. \& Illa, S., 2007, Jet noise assessment and sensitivity at aircraft level, AIAA Paper 2007-3728.

[44] Hurle, I. R., Price, R. B., Sugden, T. M. \& Thomas, A., 1968, Sound emission from open turbulent premixed flames, Proc. Roy. Soc. London, A 303, 409-427.

[45] Kaltenbacher, M., Ihme, M. \& Pitsch, H., 2007, Enhanced hybrid LES/CAA approach for combustion-induced noise in complex geometries, AIAA Paper 2007-3403.

[46] Kempton, A. J., 1976, Heat diffusion as a source of aerodynamic sound, J. Fluid Mech., 78, 1-31.

[47] Karabasov, S.A., Hynes, T.P. \& Dowling, A.P., 2007, Effect of mean-flow evolution on sound propagation through non uniform jet flows, AIAA Paper 2007-3655.

[48] Kotake, S., 1975, On combustion noise related to chemical reactions, J. Sound Vib., 42(3), 399-410.

[49] Lighthill, M.J., 1952, On sound generated aerodynamically - I. General theory, Proc. Roy. Soc. London, Vol. 211, Ser. A, 1107, 564-587.

[50] Lilley, G. M., 2003, The source of aerodynamic noise, Aeroacoustics, 2 (3 \& 4), 241-254.

[51] Lilley, G. M., 1996, The radiated noise from isotropic turbulence with applications to the theory of jet noise, J. Sound Vib., 190(3), 463-476.

[52] Lilley, G. M., 1973, On the noise from jets, proceedings of Noise mechanisms, AGARD CP-131.

[53] Lilley, G. M., 1972, The generation and radiation of supersonic jet noise. Vol. IV - Theory of turbulence generated jet noise, noise radiation from upstream sources, and combustion noise. Part II: Generation of sound in a mixing region, Air Force Aero Propulsion Laboratory, AFAPL-TR-72-53.

[54] Marble, F. E. \& Candel, S. M., 1977, Acoustic disturbance from gas nonuniformities convected through a nozzle, J. Sound Vib., 55(2), 225-243.

[55] Möhring, W., 1999, A well posed acoustic analogy based on a moving acoustic medium, in proceedings of Aeroacoustics workshop, Ed. by P. Költzsch and N. Kalitzin, 1-11. 
[56] Morfey, C. L., 1976, Sound radiation due to unsteady dissipation in turbulent flows, J. Sound Vib., 48(1), 95-111.

[57] Morfey, C. L., 1973, Amplification of aerodynamic noise by convected flow inhomogeneities, J. Sound Vib., 31(4), 391-397.

[58] Musafir, R., 2007, On the source terms in Lilley's equation, Acta Acustica United with Acustica, 93, 263-274.

[59] Muthukrishnan, M., Strahle, W.C. \& Neale, D.H., 1978, Separation of hydrodynamic, entropy, and combustion noise in a gas turbine combustor, AIAA Journal, 16(4), 320-327.

[60] NASA, 1999, Third computational aeroacoustics workshop on benchmark problems, Ohio Aerospace Institute, NASA CP-2000-209790, edited by M.D. Dahl.

[61] Oran, E. S. \& Boris, J. P., 2001, Numerical simulation of reactive flow, 2nd edition, Cambridge University Press, Cambridge.

[62] Parthasarathy, S. P., Cuffel, R. F. \& Massier, P. F., 1979, Separation of core noise and jet noise, AIAA Journal, 18(3), 256-261.

[63] Peake, N., 2004, A note on "Computational aeroacoustics examples showing the failure of the acoustic analogy theory to identify the correct noise sources" by CKW Tam, J. Comput. Acous., 12(4), 631-634.

[64] Phillips, O. M., 1960, On the generation of sound by supersonic turbulent shear layers, J. Fluid Mech., 9(1), 1-28.

[65] Pickett, G.F., 1976, Core engine noise due to turbine fluctuations convecting through turbine blade rows, Progress in Astronautics and Aeronautics, 43, 589-608.

[66] Pierce, A. D., 1990, Wave equation for sound in fluids with unsteady inhomogeneous flow, J. Acoust. Soc. Am., 87(6), 2292-2299.

[67] Plett, E. G. \& Summerfield, M., 1974, Jet engine exhaust noise due to rough combustion and nonsteady aerodynamic sources, J. Acoust. Soc. Am., 56(2), 516-522.

[68] Poinsot, T. \& Veynante, D., 2005, Theoretical and numerical combustion, 2nd edition, Edwards, Philadelphia.

[69] Pridmore-Brown, D.C., 1958, Sound propagation in a fluid flowing through an attenuating duct, J. Fluid Mech., 4, 393-406.

[70] Ramachandra, M. K. \& Strahle, W. C., 1983, Acoustic signature from flames as a combustion diagnostic tool, AIAA Journal, 21(8), 1107-1114.

[71] Rao, P. P. \& Morris, P., 2006, Use of finite element methods in frequency domain aeroacoustics, AIAA Journal, 44(7), 1643-1652.

[72] Rayleigh, J.W.S., 1877, The theory of sound, Dover Publications, New York, 2nd edition (1945), New-York.

[73] Schuller, T., Durox, D. \& Candel, S., 2002, Dynamics and noise radiated by a perturbed impinging premixed flame, Combust. Flame, 128, 88-110.

[74] Shafer, H. J., Plett, E. G. \& Summerfield, M., 1976, Optical evaluation of combustion noise source terms, AIAA Journal, 14(9), 1163-1164. 
[75] Shivashankara, B. N., Strahle, W. C. \& Handley, J. C., 1973, Combustion noise radiation by open turbulent flames, AIAA Paper 73-1025.

[76] Shivashankara, B. N., Strahle, W. C. \& Handley, J. C., 1975, Evaluation of combustion noise scaling laws by a optical technique, AIAA Journal, 13(5), 623-627.

[77] Smith, T. J. B. \& Kilham, J. K., 1963, Noise generation by open turbulent flames, J. Acoust. Soc. Am., 35(5), 715-724.

[78] Stephenson, J. \& Hassan, H.A., 1977, The spectrum of combustion generated noise, J. Sound Vib., 53(2), 283-288.

[79] Strahle, W. C., 1971, On combustion generated noise, J. Fluid Mech., 49(2), $399-414$.

[80] Strahle, W. C., 1972, Some results in combustion generated noise, J. Sound Vib., 23(1), 113-125.

[81] Tam, C.K.W., 1995, Supersonic jet noise, Annu. Rev. Fluid Mech., 27, 17-43.

[82] Thomas, A. \& Williams, G. T., 1966, Flame noise: sound emission from sparkignited bubbles of combustible gas, Proc. Roy. Soc. London, 294, 449-466.

[83] Truffaut, J.-M., Searby, G. \& Boyer, L., 1998, Sound emission by non-isomolar combustion at low Mach numbers, Combust. Theory Modelling, 2, 423-428.

[84] Wang, M., Freund, J.B. and Lele, S.K., 2006, Computational prediction of flowgenerated sound, Annu. Rev. Fluid Mech., 38, 483-512.

[85] Williams, F. A., 1985, Combustion theory, 2nd edition, Addison-Wesley Publishing Company, Readings, Massachusetts. 
